\title{
SYSTEMATIC ERRORS IN SHOT NOISE THERMOMETER MEASUREMENTS
}

\author{
Lafe Spietz ${ }^{1}$, W. Tew ${ }^{1}$, and R. J. Schoelkopf ${ }^{2}$ \\ ${ }^{1}$ NIST, ${ }^{2}$ Departments of Applied Physics and Physics, Yale University
}

\begin{abstract}
$\underline{\text { Abstract }}$
We report comparisons between the Shot Noise Thermometer (SNT), a primary thermometer based on the electronic noise from a tunnel junction, and the ITS-90 in the temperature range from $4 \mathrm{~K}$ to $30 \mathrm{~K}$. The SNT indicates a temperature that is systematically high compared to $T_{90}$, and we explain the probable origin of this effect. We characterize other systematic effects in the SNT and provide a roadmap for future extensions in the accuracy of the SNT which could make it metrologically useful in the lower-most ranges of the ITS-90 and especially in the range of the PLTS- 2000.
\end{abstract}

\section{Introduction}

In the shot noise thermometer [1], the electrical noise from a normal metal tunnel junction is measured as a function of DC bias voltage across the junction. The current spectral density of the noise from such a biased tunnel junction is

$$
S_{\mathrm{I}}(V)=2 e I \operatorname{coth}(e V / 2 k T),
$$

where $I$ is the current through the junction, $V$ is the voltage across the junction, $e$ is the charge on the electron, $k$ is Boltzmann's constant, and $T$ is temperature. By measuring the noise power in a microwave band, this curve may be quickly acquired, and temperature may be extracted as a fit parameter, independent of the gain and added noise of the measurement chain.

As long as the noise from a tunnel junction is defined by the above equation and the measurement chain is linear and voltage independent, the accuracy of the temperature measurement is determined by the accuracy of the DC voltage measurement across the junction and the precision (but not the accuracy) of the noise measurement. The systematic effects that limit the accuracy of the SNT may thus be divided into those that are due to DC voltage inaccuracies, those that are due to nonlinearities or variations in the microwave measurement chain, and those that are due to deviations of the junction noise from the above equation.

In the next section, we describe all three of these types of systematic effects and estimate their effects on the present work. The section after that will describe our recent comparisons between the SNT and a high accuracy calibrated thermometer and estimate the dominant systematic effect in these measurements. Finally, we will describe the work that would be required to improve the SNT accuracy and speculate on the future role of the SNT in temperature metrology.

\section{$\underline{\text { Systematic Effects }}$}

\section{Voltage Drops}

The first type of systematic error we will describe is inaccuracy in the DC voltage measurement across the junction. It is straightforward to measure a DC voltage on a digital multimeter to a part in $10^{5}$ without calibration and a part in $10^{6}$ with a calibration using a Zener voltage source. Thus the error in the voltage measurement is dominated by the degree to which the voltage measured by the DMM fails to accurately represent the voltage across the tunnel junction, which appears in Equation 1.

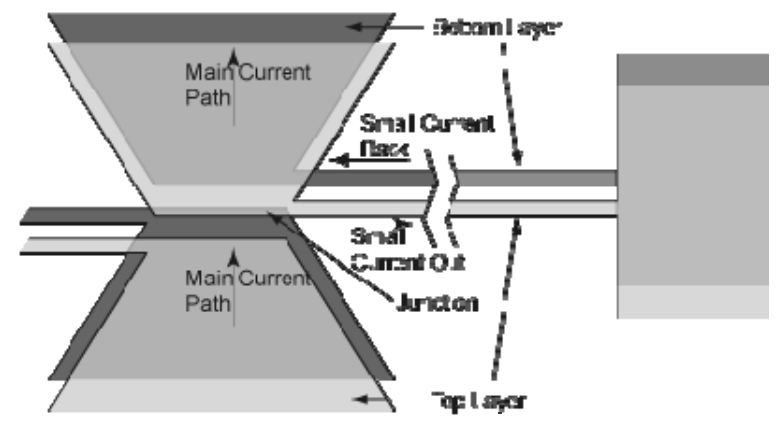

Figure 1. Cartoon of junction layout showing position of voltage and current leads, and how there can be a voltage drop within the voltage leads locally even though there is no total current in or out of the leads.

In our measurements we fabricate voltage leads as close as possible to the tunnel junction (see Fig. 1) in an attempt to minimize voltage drops in those leads, which would cause inaccuracies. Because of the double angle evaporation process used to fabricate the junctions, the voltage leads are nonideal, and they can have a voltage drop that produces to a consistently high voltage at the DMM as compared to the true voltage on the junction. Since the SNT relates temperature to measured DC voltage in a linear way, the fractional error in the voltage causes an identical fractional error in the temperature measurement. Fig. 2 shows a detailed model of our double angle evaporated junctions, which may be used to estimate the size of this effect. 


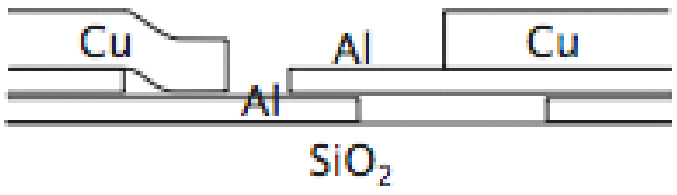

Figure 2. Schematic of overlapping layers that define junction in 3 layer Dolan bridge fabrication scheme.

The detailed estimate of the various voltage drops that contribute to the errors in temperature measurement are too complex to describe in this short a paper, so we will summarize the result here. The full analysis of voltage drops leads to a roughly constant fractional error in voltage measurement of approximately $0.4 \%$ near $4 \mathrm{~K}$. Since this voltage drop will make the voltage across the junction appear higher than it really is, this will lead to a higher inferred temperature on the SNT relative to the true temperature. Figure 3 shows the experimental comparison of the two thermometers. The increase in fractional error with increasing temperature can be explained by the changing conductance of the films.

\section{Other Systematic Effects}

For brevity in this shortened format, we will now summarize together the other systematic errors we have considered. The next largest error is that of the changing microwave impedance as a function of voltage, which changes the power coupling, causing an apparent nonlinearity in the shot noise curve. We estimate this to be a few parts in $10^{4}$ for the temperatures considered here. This effect scales with temperature because the size of conductance variations scales with $\mathrm{DC}$ voltage, and the voltage required to carry out a SNT experiment scales with temperature. Other potential systematic errors include the nonlinearity of the microwave measurement chain, quantum corrections at lower temperatures, heating of the junction, and varying leakage of microwave power into the substrate. All of these effects are either much smaller, have not yet been observed, or are relevant primarily at other temperature ranges.

\section{Experimental Comparison}

For this measurement, we constructed a cryostat specifically designed for accurate temperature comparisons between $4 \mathrm{~K}$ and room temperature, consisting of a vacuum can containing two concentric heat shields, the temperature of which was independently regulated. The vacuum can was immersed in liquid helium, and the outer heat shield was regulated at a temperature slightly above the target temperature for any given measurement. The inner shield was then regulated at the target temperature using a ceramic-encapsulated rhodiumiron resistance thermometer (RIRT) for feedback. The temperature was measured using both the SNT and a standard capsule-type RIRT, which was calibrated at NIST on the ITS-90[2]. The result of this comparison is shown in Figure 3.

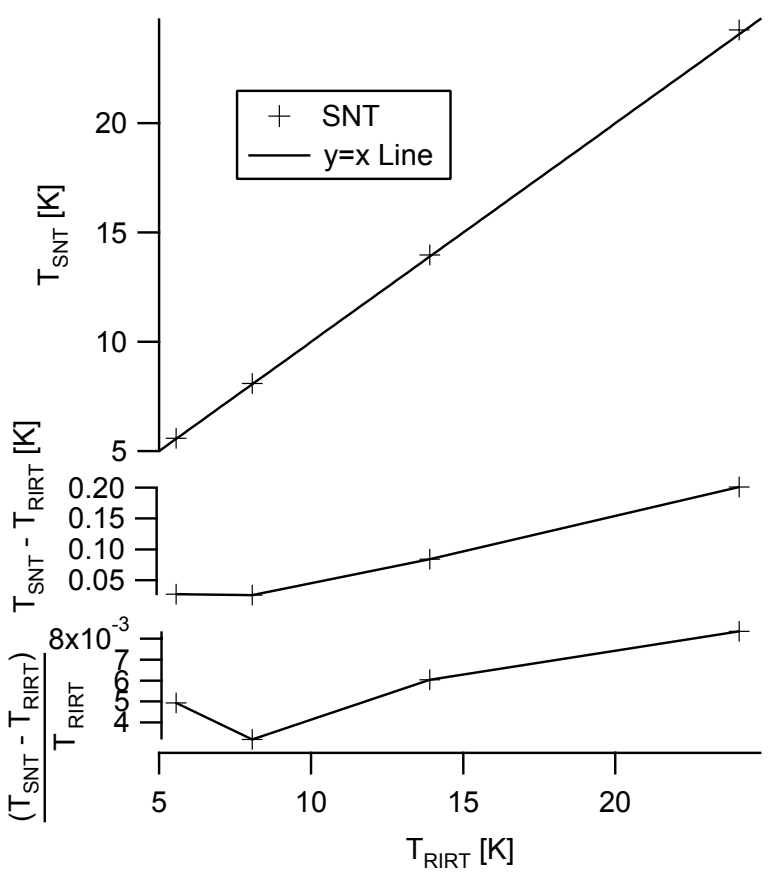

Figure 3. Temperature comparison of SNT to RIRT. The SNT always reads high relative to the more accurate thermometer.

\section{Conclusion and Future Work}

We believe that the dominant error in these SNT measurements is the voltage drop near the junction which prevents accurate measurement of the true voltage across the junction. We are in the process of designing and fabricating a new generation of shot noise thermometer junctions that use a trilayer fabrication process to reduce this error by orders of magnitude. This improvement should reduce the total error to below a part in $10^{3}$, making it a competitive standard with the PLTS-2000 in the subkelvin temperature range.

\section{$\underline{\text { References }}$}

[1] Lafe Spietz, K. W. Lehnert, I. Siddiqi, and R. J. Schoelkopf, Author, "Primary Electronic Thermometry Using the Shot Noise of a Tunnel Junction" Science, Vol. 300, no. 5627, pp. 1929-1932, June 2003.

[2] W. L. Tew and C. W. Meyer, in: Temperature: Its Measurement and Control in Science and Technology, Vol. 7, D.C. Ripple, Ed., p.p. 143-148, (2003). 